Proceedings of the

International Geometry Center

Vol. 13 , no. 3 (2020) pp. 33-48

Vol. 13, no. 3 (2020) pp. $33-48$

\title{
Heegaard diagrams and optimal Morse flows on non-orientable 3-manifolds of genus 1 and genus 2
}

\author{
Christian Hatamian, Alexandr Prishlyak
}

\begin{abstract}
The present paper investigates Heegaard diagrams of nonorientable closed 3-manifolds, i.e. a non-orienable closed surface together with two sets of meridian disks of both handlebodies. It is found all possible non-orientable genus 2 Heegaard diagrams of complexity less than 6 . Topological properties of Morse flows on closed smooth non-orientable 3-manifolds are described. Normalized Heegaard diagrams are furhter used for classification Morse flows with a minimal number of singular points and singular trajectories.
\end{abstract}

Анотація. В роботі досліджуються діаграми Хегора неорієнтовних 3многовидів. Кожна така діаграма є неорієновною замкненою поверхнею з двома наборами меридіанів на ній. Також розглядаються так звані нормалізовані діаграми, які не містять криволінійних двокутників, утворених меридіанами. Описано алгоритм, що дозволяє визначити, чи є нормалізована діаграма діаграмою Хегора деякого 3-многовиду. Діаграми Хегора роду два розбито на три типи та отримано топологічну класифікацію всіх неорієнтовних тривимірних многовидів роду два і складності не вище п'яти. Кожен такий многовид гомеоморфний зв'язній сумі лінзового простору і косого добутку 2-сфери на коло. Більш того, знайдено 3многовид складності шість, який не гомеоморфний такій сумі. Нормалізовані діаграми Хегора також використано для класифікації оптимальних потоків Морса на многовидах роду два складності не вище п'яти.

Keywords: topological equivalence, Heegaard diagram, Morse flow

Ключові слова: топологічна еквівалентність, діаграма Хегора, потік Морса DOI: http://dx.doi.org/10.15673/tmgc.v13i3.1779 


\section{INTRODUCTION}

A Heegaard diagram is a common and convenient method for representing a closed connected 3-manifold. Heegaard diagrams and Heegaard splittings are closely related. Handlebodies, Heegaard splittings and Heegaard diagrams have been studied by Y. Matsumoto in [8]. The structure of genus 2 Heegaard diagrams of orientable closed 3-manifolds has been largely discussed by S. Matveev in [9]. A simple algorithm for enumeration of orientable closed three-dimensional manifolds whose genus do not exceed two has been proposed by S. Matveev and A. Fomenko in [1]. A classification of decomposition of non-orientable 3-manifolds into 3 handlebodies with disjoint boundaries has been presented by V. Nunez, J. C. Gomezlarranaga, W. Heil in [3]. A classification of Heegaard diagrams of genus 3 has been conducted by F. Korablev in [5]. The existence of a unique non-orientable manifold of genus 1 is a consequence of W. B. R. Lickorish's result in [6]. However, in this paper, we present a certain reformulation of Lickorish's result in terms of Heegaard diagrams. Our main purpose is to investigate if there exists any non-orientable 3-manifold distinct from manifolds of type $L_{p, q} \# S^{1} \widetilde{\times} S^{2}$. Given a Heegaard diagram $H$ of $M$ there exists a polar Morse flow $X$ (only one source and only one sink) whose associated diagram is $H$. Morse flows (Morse-Smale flows without closed orbit) on manifolds with boundary have been studied by A. Prishlyak in $[12,13]$ and A. Prishlyak and A. Prus in [14], A topological classification of Morse-Smale flows on closed surfaces has been presented by M. Peiksoto in [11], V. Sharko and A. Oshemkov in [10]. Furthermore, Morse flows on three-dimensional manifolds have been studied by Ya. Umanskii in [17], A. Prishlyak in [7], Clark Robinson in [15].

\section{Heegaard Diagrams}

Let $M_{1}$ and $M_{2}$ be compact three-dimensional manifolds with homeomorphic boundaries and let $h: \partial M_{1} \rightarrow \partial M_{2}$ be any homeomorphism. Gluing the manifolds $M_{1}$ and $M_{2}$ together along this homeomorphism gives a topological space $M=M_{2} \bigcup_{h} M_{1}$.

Definition 2.1. An orientable handlebody $H$ of genus $g$ is a 3 -manifold homeomorphic to a boundary connected sum of $g$ copies of solid tori: $H=$ $\underset{i=1}{\#}\left(S^{1} \times D^{2}\right)_{i}$.

Definition 2.2. A non-orientable handlebody $H$ of genus $g$ is a 3 -manifold homeomorphic to a boundary connected sum of $g$ copies of solid Klein 
bottles:

$$
H=\underset{i=1}{g}\left(S^{1} \widetilde{\times} D^{2}\right)_{i} .
$$

Definition 2.3. A splitting of a 3-manifold $M$ into a union of two handlebodies without common interior points is called a Heegaard splitting. The genus of the splitting is the genus of the corresponding handlebodies.

Definition 2.4. Two Heegaard splittings of a 3-manifold $M$ are called equivalent if there exists a self-homeomorphism of the manifold $M$ carrying one splitting into the other.

Let $H \cup H^{\prime}$ be a Heegaard splitting of a 3-manifold $M, F=\partial H=\partial H^{\prime}$ the common boundary of genus $g$ of the two handlebodies, $u=\left\{u_{1}, \ldots, u_{g}\right\}$ the set of meridians of the handlebody $H$, and $v=\left\{v_{1}, \ldots, v_{g}\right\}$ the set of meridians of the handlebody $H^{\prime}$.

Definition 2.5. The triple $(F, u, v)$ is called a Heegaard diagram for the manifold $M$.

Conversely, let $F$ be a closed non-orientable surface of genus $g$ (Heegaard genus $g$ ) and $u=\left(u_{1}, \ldots, u_{g}\right), v=\left(v_{1}, \ldots, v_{g}\right)$ be two sets of simple closed curves on $F$ such that $u_{i} \cap u_{j}=\varnothing$ and $v_{i} \cap v_{j}=\varnothing$ for $i \neq j$. Then the triple $(F, u, v)$ is a Heegaard diagram of a certain 3-manifod iff the complements $F \backslash u$ and $F \backslash v$ are homeomorphic to 2-sphere with holes.

Definition 2.6. Two Heegaard diagrams $(F, u, v)$ and $\left(F, u^{\prime}, v^{\prime}\right)$ are called isotopic if there exists an isotopy $\varphi_{i}: F \rightarrow F$ such that $\varphi_{0}=1, \varphi_{1}(u)=u^{\prime}$ and $\varphi_{1}(v)=v^{\prime}$.

Definition 2.7. Two Heegaard diagrams $(F, u, v)$ and $\left(F, u^{\prime}, v^{\prime}\right)$ are called semi-isotopic if there exist isotopies $\varphi_{i}, \psi_{i}: F \rightarrow F$ such that $\varphi_{0}=\psi_{0}=1$, $\varphi_{1}(u)=u^{\prime}$ and $\psi_{1}(v)=v^{\prime}$.

Both operations of isotopy and semi-isotopy of Heegaard diagrams does not change the underlying 3-dimensional manifold $M$ as well as its Heegaard splitting of $M$ into handlebodies $H$ and $H^{\prime}$. Such operations only replace in $H$ and $H^{\prime}$ the meridional disks by isotopic ones.

Let $(F, u, v)$ be a Heegaard diagram and let $\beta$ be a simple curve joining the meridians $u_{1}$ and $u_{2}$ of the diagram and having no other common points with the curves of $u$. Let $C$ be a closed neighborhood of the union $u_{1} \cup u_{2} \cup \beta$ homeomorphic to a disc with two holes and intersecting no other curves of $u$. The boundary component of this neighborhood which is not isotopic to the curve $u_{1}$ or to the curve $u_{2}$, will be denoted by $u_{1} \# u_{2}$. The set $\left\{u_{1} \# u_{2}, u_{2}, \ldots, u_{g}\right\}$ will be denoted by $\tilde{u}$. 
Definition 2.8. We will say that the diagram $(F, \tilde{u}, v)$ is obtained from the diagram $(F, u, v)$ by adding $u_{2}$ to $u_{1}$ along $\beta$.

The operation of adding curves from the set $v$ is defined similarly. Notice that addition of curves from the set $u$ to curves of the set $v$ and of curves from the set $v$ to curves of the set $u$ is not allowed. The addition of one curve of a set to another curve of the same set fails not only to affect the corresponding manifold, but also its Heegaard splitting. The handlebodies of the splitting remain unchanged, and it is only their sets of meridional discs that are altered.

Definition 2.9. The diagrams $(F, u, v)$ and $\left(F, u^{\prime}, v^{\prime}\right)$ are called equivalent if we can pass from one to the other using homeomorphisms, semi-isotopies and operations of adding one meridian to another.

Proposition 2.10. If Heegaard diagrams are equivalent then the Heegaard splittings corresponding to them are equivalent.

Proof. The proof is similar to the proof of [1, Proposition 5.3]. As well as in the orientable case, the equivalence of Heegaard diagrams implies the existence of a homeomorphism between the corresponding Heegaard surfaces, which can further be extended to a homeomorphism between meridional disks, and then to a homeomorphism between 3-manifolds.

Let $(F, u, v)$ be the Heegaard diagram of a manifold $M$ corresponding to a Heegaard splitting $M=H \cup H^{\prime}$. The collections of curves $u$ and $v$ split the surface $F$ into several parts.

Suppose that a certain part is not homeomorphic to a disk. Then in this part (and, therefore, in the surface $F$ ) there exists a non-trivial simple closed curve $l$ intersecting neither the meridians $v$ of the handlebody $H^{\prime}$ nor the meridians $u$ of the handlebody $H$. Hence, this curve bounds discs in each of the handlebodies $H$ and $H^{\prime}$. The union of those disks a 2-sphere $S$ which either splits the manifold $M$ into a connected sum or gives rise to a summand $S^{2} \times S^{1}$. In both cases, the examination of the diagram reduces to the examination of one or two diagrams of lower genus.

Such a situation is therefore of no interest for us. In what follows we will assume that the curves $u$ and $v$ split the surface into disks. This happens if and only if the union $u \cup v$ is a connected graph. Such diagrams will be called connected.

So, let $(F, u, v)$ be a connected Heegaard diagram. The regions homeomorphic to disks, into which the graph $u \cup v$ cuts the surface $F$ will be interpreted as curvilinear polygons whose vertices are intersection points of meridians from different sets. Each polygon has, of course, an even number of sides. 
Definition 2.11. The Heegaard diagram $(F, u, v)$ is called normalized if among the regions into which the meridians split the surface there are no lunes.

\section{An algorithm to Determine HeegaArd diagrams}

Let $(F, u, v)$ be a normalized diagram. Curring the surface $F$ along the meridians $u=\left\{u_{1}, \ldots, u_{g}\right\}$, we will obtain a sphere with $2 g$ holes $D_{1}, \ldots, D_{g}$. The holes can be naturally divided into pairs so that each pair $D_{2 i-1}, D_{2 i}$ consists of the two holes corresponding to the meridian $u_{i}$. The meridians $v$ will then be cut into arcs joining the holes in various ways.

To recover uniquely the Heegaard diagram from such a picture, we should know how the boundary of each hole $D_{2 i}$ is glued with the boundary of its companion hole $D_{2 i-1}$. It is convenient to enumerate the points at which the meridian $u_{i}$ intersects the meridians $v$ in the order they are met when traversing the meridian $u_{i}$, and to retain these numbers in the cutting. The total number of those crossing points is called the Heegaard complexity.

We take the boundary orientations of glued holes into account by assigning a plus sign to the orientedly glued holes and a minus sign to the ones that are glued non-orientedly. In order to define the gluing maps $\varphi_{i}: \partial D_{2 i} \rightarrow \partial D_{2 i-1}, i=1,2, \ldots, g$, we introduce topological symmetries $s_{i}: \partial D_{2 i} \rightarrow \partial D_{2 i-1}$ and topological rotations $r_{i}: \partial D_{2 i-1} \rightarrow \partial D_{2 i-1}$, for $i=1,2, \ldots, g$ by the following rules:

(a) $s_{i}$ takes $\partial D_{2 i} \cap \bigcup_{i=1}^{g} v_{i}$ to $\partial D_{2 i-1} \bigcap \bigcup_{i=1}^{g} v_{i}$ so that the endpoint of each arc joining $D_{2 i}$ to $D_{2 i-1}$ is taken to the other endpoint of the same arc;

(b) $r_{i}$ shifts each point of $D_{2 i-1} \bigcap \bigcup_{i=1}^{g} v_{i}$ to the next point of $D_{2 i-1} \bigcap \bigcup_{i=1}^{g} v_{i}$.

Then an algorithm to determine Heegaard diagrams of closed 3-manifolds can be suggested as follows.

(1) An equal number of arcs should join $D_{2 i}$ and $D_{2 i-1}$.

(2) To determine how the boundaries of the holes are identified we need to enumerte the endpoints of the arcs on each of them in the cyclic order. In the case of orientedly glued holes, $D_{2 i}$ and $D_{2 i-1}$ have opposite orientation, whereas in the case of non-orientedly glued holes, $D_{2 i}$ and $D_{2 i-1}$ have the same orientation.

(3) The surface $F$ should be orientable. To verify that, we construct a dual graph for $u$ curves in $F \backslash v$. The vertices of the graph represent disjoint regions in the plane divided by the arcs of the diagram. The edges of 


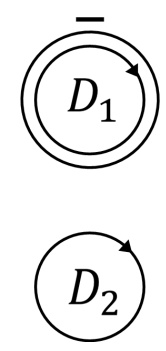

Figure 4.1. The genus 1 Heegaard diagram of a nonorientable closed 3-manifold

the graph represent the intersections of the glued holes and the regions. We assign a plus sign to each edge representing the intersection of orientedly glued holes and a minus sign to the other edges representing intersection of the ones that are glued non-orientedly. If the graph contains no cycles with odd number of minus signs, then the surface will be orientable.

(4) Every Heegaard diagram has exactly $g$ closed curves or cycles. These curves split the surface and give a sphere with $2 g$ holes which implies the surface $F$ is connected.

\section{Non-orientable HeEgaArd diagrams of Genus 1 And Genus 2}

In the case of orientable closed 3-manifolds, there are infinitely many orientable closed 3 -manifold of genus 1 , called lens spaces $L_{p, q}$, whereas in the case of non-orientable ones, the following theorem being a direct consequence of results by W.B.R. Lickorish [6] holds. We will present a proof based on Heegaard diagrams.

Theorem 4.1. ([6]) Every non-orientable closed 3-manifold of genus 1 is homeomorphic to $S^{1} \widetilde{\times} S^{2}$, see Figure 4.1 for its Heegard diagram.

Proof. According to the algorithm every genus 1 Heegaard diagram has exactly one cycle. Moreover, the surface $F$ should be orientable.

In the case of Heegaard complexity 1, there is only one possible diagram. Although this diagram has exactly one cycle. As shown in Figure 4.2 the resulting graph has a loop with a minus sign and thus the surface is not orientable. Hence there is no genus 1 Heegaard diagram of complexity 1.

In the case of Heegaard complexity 2, there are two possible diagrams as shown in Figure 4.3.

Diagram (a): if we move along the arc starting from $\left(1, D_{2}\right)$, then we will return to the starting point, since $D_{1}$ is identified with $D_{2}$, i.e. $\left(1, D_{2}\right)=$ 

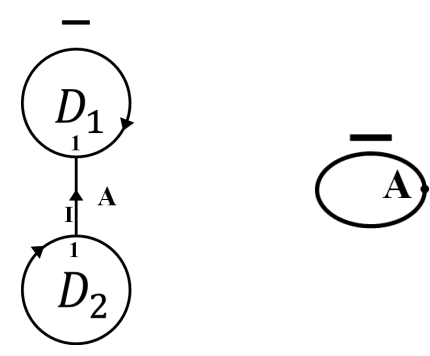

Figure 4.2. The non-orientable genus 1 diagram of complexity 1

$\left(1, D_{1}\right)$. This gives a cycle. Similarly, if we move along the arc starting from $\left(2, D_{2}\right)$, then we will return to the starting point as well, and thus we will obtain another cycle.

Thus we get a diagram with two cycles, and according to the algorithm, it is not a genus 1 Heegaard diagram.

Diagram (b): If we move along the arc starting from $\left(1, D_{2}\right)$, then we pass to $\left(2, D_{1}\right)$ which means we have arrived at $\left(2, D_{2}\right)$. Then we get to $\left(1, D_{1}\right)=\left(1, D_{2}\right)$ and once again for the same reason we return to the starting point. Thus, we ontain one cycle. As shown in Figure 4.4, although the diagram has exactly one cycle, it is not a Heegaard diagram since the resulting graph has a loop and an edge with minus signs meaning the surface $F$ is not orientable.

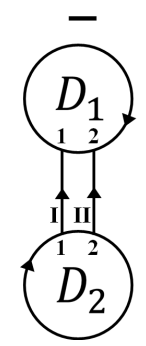

Diagram (a)

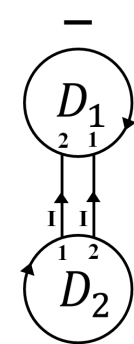

Diagram (b)

Figure 4.3. Non-orientable genus 1 diagrams of complexity 2

The total number of cycles in a non-orientable genus 1 diagram can be calculated using the following formula:

$$
\frac{P+L}{2}
$$

where $P$ is the complexity of the diagram and $L$ is the number of single-arc cycles in the diagram. If the complexity of a diagram is even, then it has 

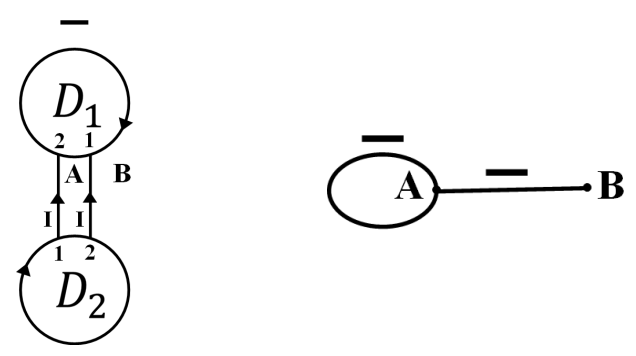

Figure 4.4. The non-orientable genus 1 diagram of complexity 1

either zero or two single-arc cycles depending on how the boundary of $D_{2}$ is glued with the boundary of $D_{1}$. In the other hand, if the complexity is odd, then it has only one single-arc cycle as shown in Figure 4.5.

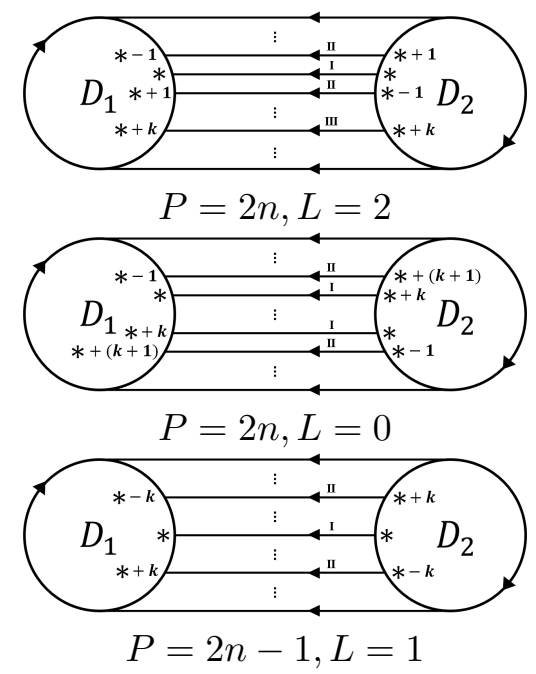

FiguRE 4.5. Non-orientable genus 1 diagrams

Formula (4.1) implies that if $P \geqslant 3$, then there are no diagrams with one cycle. This completes the proof.

Remark 4.2. In Lickorish [6] there was obtained a classification of isotopy classes of simple closed curves on the Klein bottle $K$ and proved that the mapping class group of $K$ is isomorphic to $\mathbb{Z}_{2} \times \mathbb{Z}_{2}$. One can easily deduce that any homeomorphism of a Klein bottle extends to a homeomorphism of a solid Klein bottle, which directly implies uniqueness of non-orientable closed 3-manifold of genus 1, i.e. Theorem 4.1. 
Definition 4.3. We will say that a Heegaard diagram $(F, u, v)$ of genus 2 has type I, II or III as it is depicted in Figure $4.6 a, b$ or $c$, respectively.

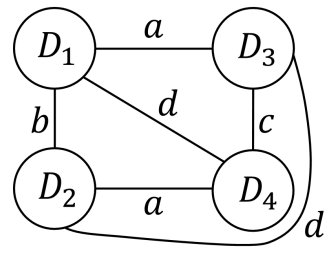

a) type I

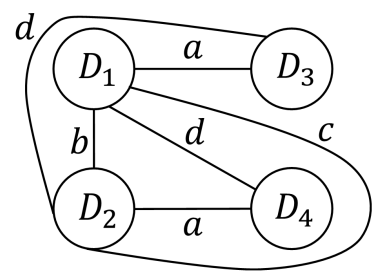

b) type II, $b, c>0$

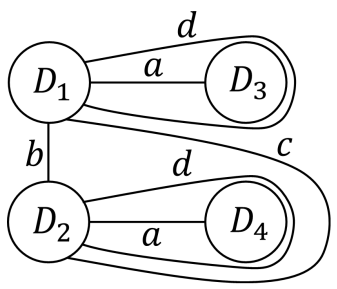

c) type III $d>0$

FiguRE 4.6. Three types of genus 2 Heegaard diagrams

Every such diagram is determined by a 8 -tuple $(t, a, b, c, d, e, f, g)$, where $t$ is the type of diagram, $a, b, c, d$ are the arcs joining holes, and $e, f$ determine the gluing maps $\varphi_{i}: \partial D_{2 i} \rightarrow \partial D_{2 i-1}, i=1,2$ and $g$ is defined to be $0,1,2$, and 3 representing $(+1,+1),(+1,-1),(-1,+1)$ and $(-1,-1)$ respectively.

Example 4.4. Let us consider the 8-tuple $(2,1,1,1,1,0,0,3)$ which represents a type II non-orientable Heegaard diagram of complexity 6 . First, we
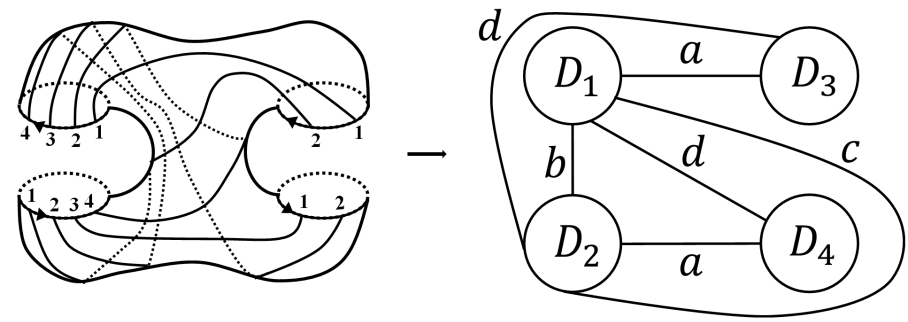

FiguRE 4.7. The diagram represented by $(2,1,1,1,1,0,0,3)$

need to make sure that the diagram shown in Figure 4.7 has exactly two closed curves.

If we move along the curve starting from $\left(2, D_{2}\right)$ in Figure 4.8 , we get to $\left(4, D_{1}\right)=\left(4, D_{2}\right)$ and then $\left(2, D_{1}\right)=\left(2, D_{2}\right)$. Again, we've ended up exactly where we started. This means this curve is closed.

Similarly, if we move along the curve starting from $\left(1, D_{2}\right)$, we eventually get to the starting point. Therefore this curve is also closed.

Having checked that the diagram has exactly two curves, we verify whether or not the surface $F$ is orientable. To do so, we construct the required graph 


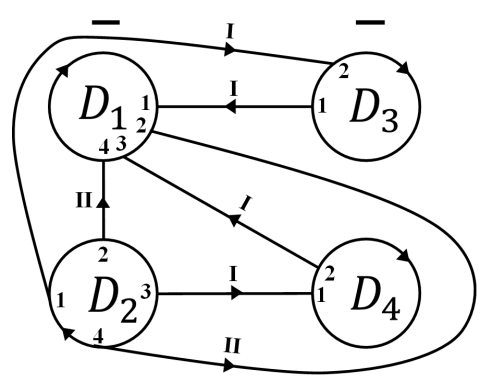

FiguRE 4.8. The cycles in the diagram represented by $(2,1,1,1,1,0,0,3)$
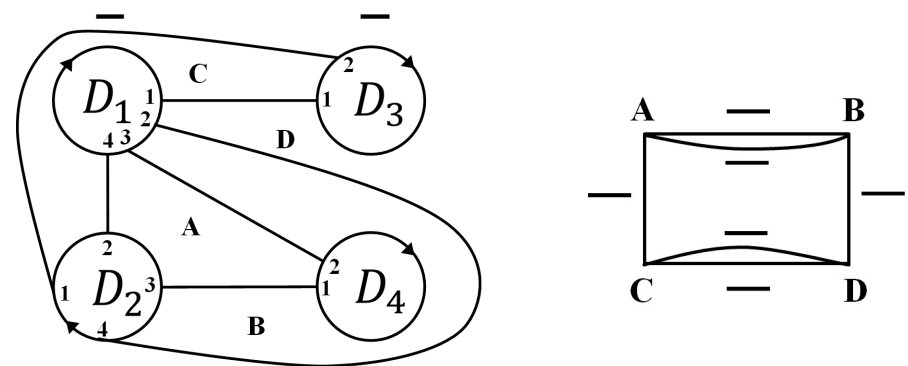

FigurE 4.9. The dual graph of $(2,1,1,1,1,0,0,3)$

according to the mappings $\varphi_{1}, \varphi_{2}$ and the boundaries orientations of the glued holes.

The resulting graph in Figure 4.9 contains no cycles with an odd number of minus signs and hence $F$ is orientable.

Finally, we need to verify whether or not the two curves split the surface. In order to do that, we construct the diagram illustrated in Figure 4.10 and make sure that we have exactly four cycles. If we move along the arc starting

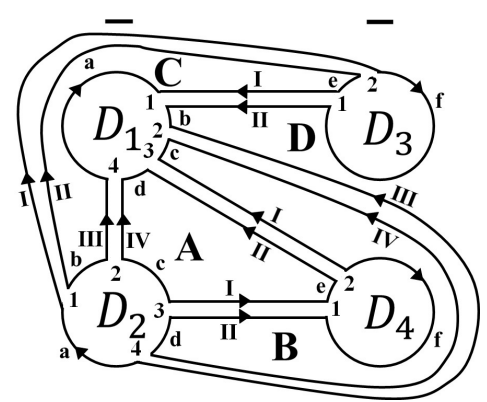

FiguRE 4.10. b-cycles of the diagram 
from the end of $\left(b, D_{2}\right)$, we get to the beginning of $\left(a, D_{1}\right)=\left(a, D_{2}\right)$. Since we have returned to the starting point we've got a cycle.

It is easy to see that the diagram in Figure 4.10 has three more cycles. Therefore the 8-tuple $(2,1,1,1,1,0,0,3)$ is a Heegaard diagram representing a certain closed non-orientable 3 -manifold.

According to [2] one can often show that two spaces are not homeomorphic by showing that their fundamental groups are not isomorphic. The fundamental group of the Heegaard diagram represented by the 8-tuple $(2,1,1,1,1,0,0,3)$ is

$$
\pi_{1}\left(M^{3}\right)=\left\langle a, b \mid a^{2}, b a b^{-1} a^{-1}\right\rangle \cong \mathbb{Z}_{2} \oplus \mathbb{Z}
$$

whereas the fundamental group of $L_{2,1} \# S^{1} \tilde{\times} S^{2}$ is

$$
\pi_{1}\left(L_{2,1} \# S^{1} \widetilde{\times} S^{2}\right) \cong \mathbb{Z}_{2} * \mathbb{Z}
$$

thus, $\pi_{1}\left(M^{3}\right) \not \pi_{1}\left(L_{2,1} \# S^{1} \widetilde{\times} S^{2}\right)$. This example shows that there exists a non-orientable Heegaard diagram of complexity 6 which is not homeomorphic to $L_{p, q} \# S^{1} \widetilde{\times} S^{2}$.

Since every genus 2 Heegaard diagram of any type has an even number of $a$ - and $d$-arcs, the following formula holds true:

$$
2(a+d)+b+c=\text { Heegaard complexity. }
$$

It can be shown that 933 non-orientable genus 2 diagrams of complexity less than 6, most of which are homeomorphic or symmetric, satisfy the equation (4.2). According to the algorithm, only 34 of them meet the criteria and represent distinct non-orientable genus 2 Heegaard diagrams. These diagrams are listed in Table 4.1.

Table 4.1: Non-orientable genus 2 Heegaard diagrams of complexity less than 6

\begin{tabular}{|c|c|c|c|c|}
\hline № & 8-tuple & Complexity & $\pi_{1}\left(M^{3}\right)$ & $\begin{array}{c}\text { Topological } \\
\text { type of } M\end{array}$ \\
\hline 1 & $(1,0,0,0,0,0,0,1)$ & 0 & $\mathbb{Z} * \mathbb{Z}$ & $S^{1} \widetilde{\times} S^{2} \# S^{1} \widetilde{\times} S^{2}$ \\
\hline 2 & $(1,0,0,0,0,0,0,3)$ & 0 & $\mathbb{Z} * \mathbb{Z}$ & $S^{1} \times S^{2} \# S^{1} \times S^{2}$ \\
\hline 3 & $(1,0,1,0,0,0,0,1)$ & 1 & $\mathbb{Z}$ & $S^{1} \times S^{2}$ \\
\hline 4 & $(1,0,2,0,0,1,0,1)$ & 2 & $\mathbb{Z}_{2} * \mathbb{Z}$ & $L_{2,1} \# S^{1} \times S^{2}$ \\
\hline 5 & $(2,0,1,1,0,1,0,1)$ & 2 & $\mathbb{Z}_{2} * \mathbb{Z}$ & $L_{2,1} \# S^{1} \times S^{2}$ \\
\hline 6 & $(3,0,0,0,1,0,0,1)$ & 2 & $\mathbb{Z} * \mathbb{Z}$ & $S^{1} \times S^{2} \# S^{1} \times S^{2}$ \\
\hline 7 & $(3,0,0,0,1,0,0,3)$ & 2 & $\mathbb{Z} * \mathbb{Z}$ & $S^{1} \times S^{2} \# S^{1} \widetilde{\times} S^{2}$ \\
\hline 8 & $(1,0,3,0,0,1,0,1)$ & 3 & $\mathbb{Z} 3 * \mathbb{Z}$ & $L_{3,1} \# S^{1} \times S^{2}$ \\
\hline
\end{tabular}


Table 4.1 - Continued from previous page

\begin{tabular}{|c|c|c|c|c|}
\hline № & 8 -tuple & Complexity & $\pi_{1}\left(M^{3}\right)$ & $\begin{array}{c}\text { Topological } \\
\text { type of } M\end{array}$ \\
\hline 9 & $(2,0,2,1,0,1,0,1)$ & 3 & $\mathbb{Z}_{3} * \mathbb{Z}$ & $L_{3,1} \# S^{1} \widetilde{\times} S^{2}$ \\
\hline 10 & $(3,0,0,1,1,0,0,1)$ & 3 & $\mathbb{Z} * \mathbb{Z}$ & $S^{1} \times S^{2} \# S^{1} \times S^{2}$ \\
\hline 11 & $(1,0,4,0,0,1,0,1)$ & 4 & $\mathbb{Z}_{4} * \mathbb{Z}$ & $L_{4,1} \# S^{1} \widetilde{\times} S^{2}$ \\
\hline 12 & $(2,0,3,1,0,1,0,1)$ & 4 & $\mathbb{Z}_{4} * \mathbb{Z}$ & $L_{4,1} \# S^{1} \times S^{2}$ \\
\hline 13 & $(2,0,2,2,0,1,0,1)$ & 4 & $\mathbb{Z}_{4} * \mathbb{Z}$ & $L_{4,1} \# S^{1} \times S^{2}$ \\
\hline 14 & $(1,2,0,0,0,0,0,3)$ & 4 & $\mathbb{Z}$ & $S^{1} \times S^{2}$ \\
\hline 15 & $(3,0,1,1,1,3,0,1)$ & 4 & $\mathbb{Z} * \mathbb{Z}$ & $S^{1} \times S^{2} \# S^{1} \times S^{2}$ \\
\hline 16 & $(3,0,2,0,1,1,0,1)$ & 4 & $\mathbb{Z} * \mathbb{Z}$ & $S^{1} \times S^{2} \# S^{1} \times S^{2}$ \\
\hline 17 & $(3,0,2,0,1,2,0,1)$ & 4 & $\mathbb{Z} * \mathbb{Z}$ & $S^{1} \times S^{2} \# S^{1} \times S^{2}$ \\
\hline 18 & $(3,0,0,0,2,1,0,1)$ & 4 & $\mathbb{Z} * \mathbb{Z}$ & $S^{1} \times S^{2} \# S^{1} \widetilde{\times} S^{2}$ \\
\hline 19 & $(3,0,0,0,2,1,0,3)$ & 4 & $\mathbb{Z} * \mathbb{Z}$ & $S^{1} \times S^{2} \# S^{1} \times S^{2}$ \\
\hline 20 & $(3,0,1,1,1,1,0,3)$ & 4 & $\mathbb{Z} * \mathbb{Z}$ & $S^{1} \times S^{2} \# S^{1} \times S^{2}$ \\
\hline 21 & $(1,0,5,0,0,1,0,1)$ & 5 & $\mathbb{Z} * \mathbb{Z}$ & $L_{5,1} \# S^{1} \times S^{2}$ \\
\hline 22 & $(1,1,0,1,1,1,0,2)$ & 5 & $\mathbb{Z}$ & $S^{1} \times S^{2}$ \\
\hline 23 & $(1,0,5,0,0,3,0,1)$ & 5 & $\mathbb{Z} * \mathbb{Z}$ & $L_{5,2} \# S^{1} \widetilde{\times} S^{2}$ \\
\hline 24 & $(2,0,4,1,0,1,0,1)$ & 5 & $\mathbb{Z} * \mathbb{Z}$ & $L_{5,1} \# S^{1} \times S^{2}$ \\
\hline 25 & $(2,0,4,1,0,2,0,1)$ & 5 & $\mathbb{Z} * \mathbb{Z}$ & $L_{5,2} \# S^{1} \widetilde{\times} S^{2}$ \\
\hline 26 & $(2,0,3,2,0,2,0,1)$ & 5 & $\mathbb{Z} * \mathbb{Z}$ & $L_{5,1} \# S^{1} \widetilde{\times} S^{2}$ \\
\hline 27 & $(2,0,3,2,0,3,0,1)$ & 5 & $\mathbb{Z} * \mathbb{Z}$ & $L_{5,2} \# S^{1} \times S^{2}$ \\
\hline 28 & $(3,1,0,1,1,1,0,2)$ & 5 & $\mathbb{Z}$ & $S^{1} \widetilde{\times} S^{2}$ \\
\hline 29 & $(3,0,3,0,1,1,0,1)$ & 5 & $\mathbb{Z} * \mathbb{Z}$ & $S^{1} \times S^{2} \# S^{1} \widetilde{\times} S^{2}$ \\
\hline 30 & $(3,0,3,0,1,2,0,1)$ & 5 & $\mathbb{Z} * \mathbb{Z}$ & $S^{1} \times S^{2} \# S^{1} \widetilde{\times} S^{2}$ \\
\hline 31 & $(3,0,2,1,1,4,0,1)$ & 5 & $\mathbb{Z} * \mathbb{Z}$ & $S^{1} \times S^{2} \# S^{1} \widetilde{\times} S^{2}$ \\
\hline 32 & $(3,0,1,2,1,4,0,1)$ & 5 & $\mathbb{Z} * \mathbb{Z}$ & $S^{1} \times S^{2} \# S^{1} \widetilde{\times} S^{2}$ \\
\hline 33 & $(3,0,1,0,2,1,0,1)$ & 5 & $\mathbb{Z} * \mathbb{Z}$ & $S^{1} \times S^{2} \# S^{1} \times S^{2}$ \\
\hline 34 & $(3,0,1,0,2,2,0,1)$ & 5 & $\mathbb{Z} * \mathbb{Z}$ & $S^{1} \times S^{2} \# S^{1} \widetilde{\times} S^{2}$ \\
\hline & & & & \\
\hline
\end{tabular}

Theorem 4.5. If the Heegaard complexity of a closed non-orientable 3manifold $M$ is $\leqslant 5$, then $M$ is homeomorphic to $L_{p, q} \# S^{1} \tilde{\times} S^{2}$.

Proof. Table 4.1 gives information about each genus 2 Heegaard diagram of complexity less than 6 .

We present the proof only for Heegaard complexity 1 as the other cases $2 \leqslant$ complexity $\leqslant 5$ can be proved analogously.

In this case, using formula (4.2) we have

$$
2(a+d)+b+c=1 .
$$


Here are the possible 8-tuples which satisfy formula (4.3)

$$
\begin{aligned}
& \{(1,0,1,0,0,0,0,1),(1,0,1,0,0,0,0,2),(1,0,1,0,0,0,0,3) \\
& (1,0,0,1,0,0,0,1),(1,0,0,1,0,0,0,2),(1,0,0,1,0,0,0,3)\} .
\end{aligned}
$$

Since clearly

$$
\begin{aligned}
& (1,0,1,0,0,0,0,1) \cong(1,0,0,1,0,0,0,1), \\
& (1,0,1,0,0,0,0,2) \cong(1,0,0,1,0,0,0,2), \\
& (1,0,1,0,0,0,0,3) \cong(1,0,0,1,0,0,0,3),
\end{aligned}
$$

it's enough to consider the diagrams represented by

$$
(1,0,1,0,0,0,0,1), \quad(1,0,1,0,0,0,0,2), \quad(1,0,1,0,0,0,0,3) .
$$

As Figure 4.11 indicates, the resulting graph of the diagram represented by $(1,0,1,0,0,0,0,1)$ has no cycle with minus sign and thus the surface $F$ is orientable. Moreover, the diagram has two closed curves. Therefore $(1,0,1,0,0,0,0,1)$ represents a Heegaard diagram.
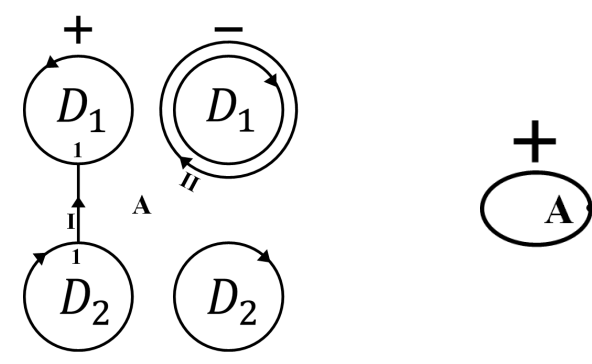

FiguRE 4.11. The dual graph of $(1,0,1,0,0,0,0,1)$

The resulting graphs of the diagrams represented by $(1,0,1,0,0,0,0,2)$ and $(1,0,1,0,0,0,0,3)$ are identical and both have a cycle with minus sign which implies that the surface $F$ is not orientable. Therefore they do not represent Heegaard diagrams.

\section{Optimal Morse flows on 3-MANifolds}

A vector field (and the corresponding flow) on a closed manifolds is called a Morse if the following conditions hold true:

(1) it has finitely many singular points which are non-degenerate;

(2) stable and unstable manifolds of singular points intersects transversely;

(3) $\alpha$ - and $\omega$-limit sets of each trajectory are singular points. 
Let $M$ be a smooth closed 3-manifold. We construct a Morse flow diagram which is in the form of a surface and two sets of circles embedded into it.

A surface $F$ is the boundary of the regular neighborhood of the union of sources and stable manifolds with singular points of Morse index 1. On the surface $F$ we choose the following sets of closed curves:

(1) curves $u$, which are intersections of unstable manifolds with singular points of Morse index 1 and the surface $F$;

(2) curves $v$, which are intersections of stable manifolds with singular points of Morse index 2 and the surface $F$.

By a surgery on $F$ along $u$ we obtain a 2 -sphere with holes which its boundary component corresponds to the boundary of singular points, i.e. one source and several saddles.

A Morse flow diagram on a three-dimensional manifold is the 3-tuple $(F, u, v)$ consisting of a surface $F$ and two sets of simple closed curves $u$ and $v$ on it.

Two Morse flow diagrams are said to be equivalent if there is a surface homeomorphism that maps sets of curves into sets of curves of the same type.

According to [12], two Morse flows on 3-manifold are topologically equivalent if and only if their diagrams are isomorphic.

A Morse flow is called optimal if it has the least number of singular points and trajectories between the saddles.

Lemma 5.1. A Morse flow is optimal if and only if it contains only one sink and one source.

Proof. A proof of this theorem in dimension 2 can be found in [4]. For arbitrary dimension greater than 1, it can be proved by the correspondence between Morse flows and Morse functions discussed in [16] and the well-known fact from Morse theory that any Morse function on a closed connected manifold with minimal number of critical points has one local minimum and one local maximum.

An optimal (polar) Morse flow diagram on a closed 3-manifold is similar to a Heegaard diagram.

Theorem 5.2. There exist

- a unique optimal Morse flow on $S^{1} \tilde{\times} S^{2}$ up to topological equivalence,

- 2 optimal Morse flows on $S^{1} \tilde{\times} S^{2} \# S^{1} \tilde{\times} S^{2}$ up to topological equivalence,

- 2 flows on $L_{2,1} \# S^{1} \tilde{\times} S^{2}$ up to topological equivalence,

- 2 flows on $L_{3,1} \# S^{1} \tilde{\times} S^{2}$, which are not topologically equivalent, 
- 3 flows on $L_{4,1} \# S^{1} \tilde{\times} S^{2}$,

- 3 flows on $L_{5,1} \# S^{1} \tilde{\times} S^{2}$,

- 3 flows on $L_{5,2} \# S^{1} \widetilde{\times} S^{2}$.

There are no other optimal flows on closed non-orientable 3-manifolds of complexity less than 6.

Proof. Optimal Morse flow diagrams on $S^{1} \widetilde{\times} S^{2}$ are of complexity 0. This implies that diagrams $3,14,22$, and 28 in Table 4.1 are not optimal. The following diagrams in Table 4.1 give optimal Morse flows:

- diagram 1 on $S^{1} \tilde{\times} S^{2} \# S^{1} \tilde{\times} S^{2}$

- diagram 2 on $S^{1} \tilde{\times} S^{2} \# S^{1} \tilde{\times} S^{2}$

- diagrams 4 and 5 on $L_{2,1} \# S^{1} \widetilde{\times} S^{2}$

- diagrams 8 and 9 on $L_{3,1} \# S^{1} \widetilde{\times} S^{2}$

- diagrams 11,12 and 13 on $L_{4,1} \# S^{1} \tilde{\times} S^{2}$

- diagrams 21,20 and 26 on $L_{5,1} \# S^{1} \widetilde{\times} S^{2}$

- diagrams 23,25 and 27 on $L_{5,2} \# S^{1} \widetilde{\times} S^{2}$

All other diagrams from Table 4.1 give non-optimal Morse flows as they have more intersections (saddle connections) than the above diagrams.

\section{Conclusion}

We have shown that if the Heegaard complexity of a non-orientable closed 3 -manifold $M$ is $\leqslant 5$, then $M$ is homeomorphic to $L_{p, q} \# S^{1} \widetilde{\times} S^{2}$.

It turns out that there exists a non-orientable Heegaard diagram of complexity 6 which is not homeomorphic to $L_{p, q} \# S^{1} \widetilde{\times} S^{2}$.

Our findings indicate that there is a bijection (up to homeomorphism) between Heegaard diagrams and polar Morse flows on 3-manifolds (up to topological equivalence).

\section{REFERENCES}

[1] A. T. Fomenko, S. V. Matveev. Algorithmic and computer methods for three-manifolds, volume 425 of Mathematics and its Applications. Kluwer Academic Publishers, Dordrecht, 1997, doi: 10.1007/978-94-017-0699-5. Translated from the 1991 Russian original by M. Tsaplina and Michiel Hazewinkel and revised by the authors, With a preface by Hazewinkel.

[2] Allen Hatcher. Algebraic topology. Cambridge University Press, Cambridge, 2002.

[3] Wolfgang Heil, Víctor Núñez, J. C. Gómez-Larrañaga. Stiefel-Whitney surfaces and the tri-genus of non-orientable 3-manifolds. Manuscripta Math., 100(4):405-422, 1999, doi: $10.1007 / \mathrm{s} 002290050209$.

[4] Zlata Kibalko, Alexandr Prishlyak, Roman Shchurko. Trajectory equivalence of optimal Morse flows on closed surfaces. Proc. Int. Geom. Cent., 11(1):12-26, 2018, doi: $10.15673 /$ tmgc.v11i1.916.

[5] F. Korablëv. Classification of Heegaard diagrams of genus 3. Fundam. Prikl. Mat., 11(5):91-97, 2005, doi: 10.1007/s10958-007-0364-2. 
[6] W. B. R. Lickorish. Homeomorphisms of non-orientable two-manifolds. volume 59, pages 307-317, 1963, doi: 10.1017/s0305004100036926.

[7] D. P. Lychak, A. O. Prishlyak. Morse functions and flows on nonorientable surfaces. Methods Funct. Anal. Topology, 15(3):251-258, 2009.

[8] Yukio Matsumoto. An introduction to Morse theory, volume 208 of Translations of Mathematical Monographs. American Mathematical Society, Providence, RI, 2002, doi: $10.1090 / \mathrm{mmono} / 208$. Translated from the 1997 Japanese original by Kiki Hudson and Masahico Saito, Iwanami Series in Modern Mathematics.

[9] Sergei Matveev. Algorithmic topology and classification of 3-manifolds, volume 9 of Algorithms and Computation in Mathematics. Springer-Verlag, Berlin, 2003, doi: 10.1007/978-3-662-05102-3.

[10] A. A. Oshemkov, V. V. Sharko. On the classification of Morse-Smale flows on two-dimensional manifolds. Mat. Sb., 189(8):93-140, 1998, doi: 10.1070/SM1998v189n08ABEH000341.

[11] M. M. Peixoto. Structural stability on two-dimensional manifolds. Topology, 1:101-120, 1962, doi: 10.1016/0040-9383(65)90018-2.

[12] Alexandr Prishlyak. Complete topological invariants of morse-smale flows and handle decompositions of 3-manifolds. Journal of Mathematical Sciences, 144(5):4492-4499, 2007.

[13] Alexandr Prishlyak. Topological equivalence of Morse-Smale vector fields on 3manifolds. Talk at the International conference "Contemporary problems in the Theory of dynamical systems", Nigniy Novgorod, July, 1996.

[14] Alexandr Prishlyak, Andrei Prus. Topological properties of Morse-Smale flows on a compact surface with boundary. Talk at the International conference "Morse theory and its applications" dedicated to the memory and 70th anniversary of Volodymyr Sharko (25.09.1949-07.10.2014), Kyiv, Ukraine, September 25-28, 2019, https://www. imath.kiev.ua/ topology/conf/sharko70/contents/sharko70_abstracts.pdf.

[15] Clark Robinson. Structural stability on manifolds with boundary. J. Differential Equations, 37(1):1-11, 1980, doi: 10.1016/0022-0396(80)90083-2.

[16] Stephen Smale. On gradient dynamical systems. Ann. of Math. (2), 74:199-206, 1961, doi: $10.2307 / 1970311$.

[17] Ya. L. Umanskiř. Necessary and sufficient conditions for topological equivalence of three-dimensional dynamical Morse-Smale systems with a finite number of singular trajectories. Mat. Sb., 181(2):212-239, 1990, doi: 10.1070/SM1991v069n01ABEH001235.

Received: July 7, 2020, accepted: September 20, 2020.

Christian Hatamian

Institute of Mathematics NAS of Ukraine, 3, Str. Tereschenkivska, Kyiv, 01024

UKRAINE

Email: christian1.618@icloud.com

Alexandr Prishlyak

Taras Shevchenko National University of Kyiv, 64/13, Str. Volodymyrska, KYIV, UKRAINE

Email: prishlyak@yahoo.com

ORCID: orcid.org/0000-0002-7164-807X 